Published in final edited form as:

Neuropsychol Rev. 2011 June ; 21(2): 73-80. doi:10.1007/s11065-011-9166-x.

\title{
Fetal Alcohol Spectrum Disorders: An Overview
}

\author{
Edward P. Riley, \\ Department of Psychology, Center for Behavioral Teratology, San Diego State University, 6330 \\ Alvarado Court, Suite 100, San Diego, CA 92120, USA
}

M. Alejandra Infante, and

Department of Psychology, Center for Behavioral Teratology, San Diego State University, 6330

Alvarado Court, Suite 100, San Diego, CA 92120, USA

San Diego State University/University of California San Diego, Joint Doctoral Program in Clinical Psychology, San Diego, CA, USA

\section{Kenneth R. Warren}

National Institute on Alcohol Abuse and Alcoholism, Bethesda, MD, USA

\section{Abstract}

When fetal alcohol syndrome (FAS) was initially described, diagnosis was based upon physical parameters including facial anomalies and growth retardation, with evidence of developmental delay or mental deficiency. Forty years of research has shown that FAS lies towards the extreme end of what are now termed fetal alcohol spectrum disorders (FASD). The most profound effects of prenatal alcohol exposure are on the developing brain and the cognitive and behavioral effects that ensue. Alcohol exposure affects brain development via numerous pathways at all stages from neurogenesis to myelination. For example, the same processes that give rise to the facial characteristics of FAS also cause abnormal brain development. Behaviors as diverse as executive functioning to motor control are affected. This special issue of Neuropsychology Review addresses these changes in brain and behavior highlighting the relationship between the two. A diagnostic goal is to recognize FAS as a disorder of brain rather than one of physical characteristics.

\section{Keywords}

Fetal alcohol syndrome (FAS); Fetal alcohol spectrum disorders (FASD); Prenatal alcohol exposure; Brain-behavior

\begin{abstract}
Although there are historical references dating from Aristotle and the Bible regarding the detrimental effects of prenatal alcohol exposure (see Calhoun and Warren 2007 for a review), the modern advent of the study of fetal alcohol syndrome (FAS) and related conditions began with the publication of two papers in 1973 (Jones and Smith 1973; Jones et al. 1973). In those two papers a pattern of birth anomalies resulting from prenatal alcohol exposure was described and the term "fetal alcohol syndrome" was introduced to the medical community (Jones and Smith 1973). Since this initial description of FAS, over 3500 papers have been published on the topic, as demonstrated by a PubMed search using the term "fetal alcohol syndrome." While there are still many unanswered questions, several
\end{abstract}

C) Springer Science+Business Media, LLC 2011

eriley@mail.sdsu.edu.

Disclosure The authors have no financial relationships to disclose. 
facts have become clear: 1) alcohol is a teratogen that can have devastating effects on the developing embryo and fetus; 2) the range of outcomes following gestational alcohol exposure is broader than those described as FAS; 3 ) the most profound effects of gestational alcohol exposure are those it has on cognitive and behavioral outcomes and on brain development; and 4) the consequences of prenatal alcohol exposure represent a major public health issue, having wide ranging effects at a staggering economic cost.

\section{Terminology}

In their original reports, Jones and colleagues (Jones and Smith 1973, Jones et al. 1973, Jones et al. 1976) described the features shared by a relatively small group of infants and children born to women who were clinically recognized as having an alcohol use disorder during the time frame of their pregnancy. The characteristics observed in the 11 initial cases included pre and postnatal growth retardation (100\%), developmental delay or mental deficiency (100\%), and distinct craniofacial anomalies, including short palpebral fissures $(100 \%)$ and maxillary hypoplasia (64\%), joint and palmar crease anomalies (73\%), and cardiac defects $(70 \%)$.

Over the years the diagnosis of FAS has been expanded and refined but still includes many of the original anomalies found in these original cases. Currently, there are four commonly used diagnostic schemas, which are summarized in Table 1 (Astley and Clarren 2000 (4digit code); Bertrand et al. 2004, 2005 (National Task Force/CDC); Chudley et al. 2005 (Canadian Guidelines); Hoyme et al. 2005 (Revised IOM)). Despite differences among them, all four schemas rely on anomalies in three distinct areas; prenatal and/or postnatal growth deficiency; central nervous system (CNS) dysfunction; and a characteristic pattern of facial anomalies.

The different diagnostic schemas all agree on the facial characteristics used to define FAS but differ in how many must be present to obtain a diagnosis. The most distinctive facial characteristics are short palpebral fissures, a smooth philtrum, and a thin upper vermillion border (Fig. 1). In all four schemas, growth deficiency for FAS is defined as pre or postnatal weight or height at or below the 10th percentile. The CNS dysfunction has the broadest range of potential deficits and is the most variable among the different schemas, but is most consistently defined as evidence of a structural brain anomaly, head circumference below the 10th percentile, or evidence of pervasive neurological or other CNS dysfunction.

Soon after the identification of FAS it became clear that not all individuals with heavy prenatal alcohol exposure demonstrated all of the features required for a diagnosis. The range of physical and behavioral outcomes was variable, and animal experimentation identified a number of factors that could influence the outcome, including the dose, timing of exposure, genetic factors, and nutritional status of the mother. The term "suspected fetal alcohol effects" (FAE) was suggested to deal with the less complete or partial expression of the syndrome (Clarren and Smith 1978), but because of a lack of precision and its frequent misuse, it was subsequently recommended that the term be discontinued (Aase et al. 1995). Two years later the Institute of Medicine (IOM) (Stratton et al. 1996) suggested new terminology to encompass the range of consequences following prenatal alcohol exposure. First, there was FAS, with or without confirmed maternal alcohol exposure. It was felt that the criteria for a diagnosis of FAS were sufficiently distinct and specific that the diagnosis could be made in the absence of knowledge about the history of alcohol exposure. The IOM also suggested the term Partial FAS (pFAS) when there was a confirmed history of prenatal alcohol exposure and some components of the full syndrome but not enough to establish the diagnosis of FAS. Further, because some of the characteristic features of FAS may change over time (e.g., facial features, growth deficits), this category allowed for someone with a 
subset of features to be recognized as having an alcohol-related effect. Consequently, the IOM recommended use of the terms "Alcohol Related Birth Defects" (ARBD) and "Alcohol Related Neurodevelopmental Disorder" (ARND). These categories were added to ensure inclusion of physical or behavioral disorders or conditions where previous clinical or animal studies had linked gestational alcohol exposure to these outcomes. ARND was more formally defined by Hoyme et al. (2005) and Chudley et al. (2005). Both schemas allowed for this terminology when a complex pattern of behavioral and cognitive abnormalities are noted that could not be otherwise explained, and when there is known significant maternal alcohol use. Both also provide examples of the type of problems that could be related to alcohol exposure.

Given the wide range of effects resulting from prenatal alcohol exposure, the term "fetal alcohol spectrum disorders" (FASD) came into use (Bertrand et al. 2005; Sokol, et al. 2003). FASD is a non-diagnostic umbrella term identifying the range of outcomes from gestational alcohol exposure. Thus, FAS, pFAS, ARND, and ARBD would all be subsumed under the FASD umbrella. Additionally, it could also include spontaneous abortion (Kline et al. 1980; Harlap and Shiono 1980), sudden infant death (Burd et al. 2004), or any other condition thought to be related to prenatal alcohol exposure.

\section{Epidemiology}

The Center for Disease Control and Prevention (CDC) acknowledges a wide range of estimates for FASD, but estimates from their early studies (Center for Disease Control and Prevention 1993, 1995, 1997, 2002) range between 0.2 to 1.5 cases per 1000 live births. At about the same time, May and Gossage (2001) estimated the prevalence of FAS to be 0.5 to 2 cases per 1000 births. When considering the full range of prenatal alcohol effects, the estimated prevalence of FASD escalated to approximately 1 per $100(1 \%)$ live births (Sampson et al. 1997). More recently, May et al. (2009) evaluated active case ascertainment studies with an emphasis on in-school studies and estimated that in a typical, mixed racial and mixed socioeconomic population in the U.S., the prevalence of FAS may be 2-7 per 1000. When considering FASD, they estimated the prevalence as high as $2-5 \%$ of younger school children in the US and Western Europe. The highest rates of FAS and partial FAS have been reported from South Africa where alarming rates between 68.0 and 89.2 per 1000 have been found in a mixed race population with a historically high prevalence of risk drinking (May et al. 2007). Other countries also report fairly high prevalence rates of FASD. For example, in Croatia the estimated prevalence of FAS is 6.44 per 1000 and 34.3 per 1000 for pFAS (Petković and Barisić 2010). In the Lazio region of Italy the rate of FAS was 3.7 to 7.4 per 1000 children and the rate of FASD was 20.3 to 40.5 per 1000 (May et al. 2006). This would mean that between 2.3 and $4.1 \%$ of all children in this region are affected by prenatal alcohol exposure. Obviously, additional surveillance studies are needed to sort out the true prevalence of FASD in various populations, but even conservative estimates provide reason for serious concern. Recently, the World Health Organization convened a meeting to establish procedures for a surveillance study based upon in-school screeners, with a focus on developing countries.

\section{Biomarkers}

There are also data available on alcohol exposure using biomarkers germane to this discussion. When alcohol is metabolized, fatty acid ethyl esters (FAEEs) accumulate in the meconium, the first stool passed by the newborn, and this non-oxidative metabolite can be a marker of second and third trimester alcohol exposure. In assessing FAEEs in meconium from over 900 births in Uruguay, Hutson et al. (2010) found that 44\% were positive for alcohol exposure above a set cutoff ( $>2 \mathrm{nmol} / \mathrm{g}$ ), which the authors believe represents an "at 
risk" level of exposure. In Barcelona, Spain $45 \%$ of the meconium samples exceeded this cutoff (Garcia-Algar et al. 2008). In Grey Bruce, Canada, 17 (2.5\%) of 682 meconium samples tested positive for high levels of exposure, which was 5 times greater than the number of high risk exposures detected via questionnaire (Gareri et al. 2008). In a follow-up study of "high risk" obstetrical patients, $30 \%$ tested positive for high levels of FAEEs (Goh et al. 2010). Although more research needs to be done on the usefulness of FAEEs as a biomarker for prenatal alcohol exposure (Burd and Hofer 2008), this test has been proposed as a cost-effective means to identify high-risk offspring (Gifford et al. 2010). However, research to develop other biomarkers of maternal alcohol use also needs to be pursued for several reasons. First, any meconium assay will have the limitation of providing information on maternal drinking only after childbirth, and even then only for the latter two trimesters.

An alternative approach would be to measure FAEEs in another tissue such as maternal hair (Kulaga et al. 2010). In addition, other research is on-going on a range of other biomarkers, such as micro-RNA, and potential proteomic and metabolic markers (Datta et al. 2008).

\section{Cost}

Cost estimates of FAS in the U.S. have increased over the years. In 1991 the annual cost of FAS was estimated to be approximately $\$ 75$ million (Abel and Sokol 1991). In 2002 the estimated annual cost of FAS was thought to reach $\$ 3.6$ billion (Lupton et al. 2004). A Canadian study reported the annual cost per individual with FASD to be $\$ 21,642$, and the overall estimated annual cost for FASD at $\$ 5.3$ billion (Stade et al. 2009). Most recently, the U.S. National Task Force on FAS reported the annual cost of FAS in the U.S. to be \$3.6 billion (Olson et al. 2009), with the cost associated with FASD to be much higher. Based simply on cost, it is clear that FASD is a major public health problem.

\section{The effects of prenatal alcohol exposure on brain and behavior}

Studies involving animal model systems clearly show that prenatal alcohol exposure affects all stages of brain development from neurogenesis to myelination, through a variety of mechanisms, including disrupted cell-cell interactions, altered gene expression, oxidative stress, and growth factor signaling disruptions (see Goodlett et al. 2005 for a review). As noted, the most profound effects of prenatal alcohol exposure disrupt brain development and subsequent cognitive, motor, and behavioral functions. The majority of articles in this special issue of the journal review that evidence. In the first paper, Mattson et al. provide a review of the neuropsychological and behavioral consequences of prenatal alcohol exposure. Of particular importance is the evidence that similar findings are seen in children with FAS and those who might be labeled as ARND. This is relevant for identifying a "behavioral profile or phenotype" of prenatal alcohol exposure, given that so many individuals with an ARND fall through the cracks in systems of care. To identify the full range of outcomes resulting from gestational alcohol exposure, we need to define the behavioral characteristics resulting from such exposure. This is indeed a challenging task, and more than one behavioral phenotype might be required, given differences in the risk factors for FASD. This article is also important for laying down the foundation for understanding the brainbehavioral relationships assessed in the other articles. Some of these brain-behavior relationships are pointed out in the article by Lebel et al. in which structural MRI studies indicate widespread abnormalities in brain following gestational alcohol exposure. Of significant interest is that in some cases the extent of structural brain abnormalities correlates with severity of FAS facial features, and more pronounced FAS facial characteristics are associated with more severe behavioral problems.

The remaining articles focus on specific aspects of brain and behavior in FASD, where data are more limited. The article by Coles and Li reviews findings based on functional and 
biochemical neuroimaging methods. These are recent reports and illustrate the feasibility of using neuroimaging in this population. A notable refrain in the functional studies is the identification of compromised efficacy in the processing resources of the brain. Despite inconsistencies in the published literature, the authors note the limitations of the studies in this burgeoning field and discuss variables and confounds that may have influenced the outcomes.

White matter deficits have been noted for some time in individuals with a history of prenatal alcohol exposure, and Wozniak and Muetzel pursue this finding with a review of the microstructural integrity of white matter using diffusion tensor imaging (DTI). Again, the history of this method in research on FASD is relatively brief, but several studies have identified abnormalities in white matter integrity, especially in the corpus callosum, a structure affected by prenatal alcohol exposure (see Lebel et al. this volume). Recent whole brain DTI studies have reported widespread disruption of integrity and, importantly, relationships between white matter integrity and behavior have been noted. Of interest, the authors consider the findings in FASD with those of other disorders.

As discussed in the Mattson et al article (this volume), the identification of a behavioral phenotype related to prenatal alcohol would be a major advance in assisting with the diagnosis of the full range of effects from prenatal alcohol exposure. A similar theme is discussed in the paper by Jacobson et al., where the authors propose the use of specific cognitive measures to assist in identifying individuals prenatally exposed to alcohol. Children with histories of in utero alcohol exposure show specific deficits in magnitude comparisons and in eyeblink conditioning. The authors review these studies and draw relationships to the underlying brain mechanisms involved in these behaviors observed in individuals with FASD. Of importance, because the neural mechanisms underlying these behaviors are known, they can be assessed early in life to identify potentially high-risk infants and may also be useful in determining the influence of therapeutic interventions.

Many of the significant advances for understanding FASD have been provided by animal models of this disorder. There is a good degree of concordance between the findings with these animal models and with the clinical condition. In fact, these animal models played a critical role in establishing both the teratogenicity and the behavioral teratogenicity of alcohol. Their use in assessing both behavioral and brain effects is an excellent example of the translational nature of research in this field. The animal models were instrumental in controlling for many confounds present in the clinical work and for identifying intervening variables. O'Leary-Moore et al. provide a review of the use of imaging in a mouse model of FASD, which serves as an excellent complement to the human studies already reviewed. The imaging methods used in animals have the potential to yield new insights into human condition, to provide a valuable adjunct to the human studies in terms of confirmatory results, and to discover developmentally specific effects. They are proving useful in identifying brain anomalies not previously described in humans, showing the relationship between facial dysmorphology and brain structure, and as methods improve and are translated to the human condition should be valuable additions to assessing potential interventions.

Animal model findings are also presented in the article by Schneider et al., which presents an overview of the rodent animal model data and then describes findings from one of the few primate models of FASD. What is perhaps most compelling in this model is that the dose of alcohol used produces blood alcohol levels in the moderate range, while showing both brain and behavioral data consistent with the rodent data in which higher blood alcohol levels are often used and the human data where the exposure data is often unavailable or 
inaccurate. Furthermore, this model includes a stress component, which is relevant given that early stress may be a significant contributing factor in FASD.

One of the issues raised in several of the papers in this issue involve the early identification of individuals with histories of prenatal alcohol exposure, particularly those without the facial features of FAS. Early identification is necessary in order to provide interventions at the earliest possible time. The last article in this series by Kodituwakku and Kodituwakku present an overview of the intervention efforts in children with an FASD and preclinical work in this area. Although there is reason to assume that interventions proven effective in other disorders would also be effective in FASD, this paper presents a dynamic interactive framework combining various interventions in order to design a strategy to treat individuals with an FASD.

\section{Summary}

Nearly 40 years of research have demonstrated that alcohol is a potent physical and behavioral teratogenic agent. While the debate rages on about whether or how much alcohol during pregnancy is safe or whether there are periods during pregnancy when it might be safe to drink, the data clearly show that alcohol can have devastating effects on brain and behavioral development. These effects of gestational alcohol exposure are by far the most profound, having life-long implications for the affected individual, the family, and society. Individuals with histories of prenatal alcohol exposure are at risk for problems in mental health, school, the justice system, and independent living in general (e.g., Spohr and Steinhausen 2008). The articles on FASD in this issue review what we know in terms of brain and behavioral outcomes in these individuals. Although the data in many cases are limited given that this field is still young, they present a compelling case for the underlying CNS cause of the many problems faced by these individuals. As technologies become more refined, better methodologies for identifying these individuals will become available. One major challenge is determining means for differential diagnosis of individuals with FASD relative to those with other disorders yet similar symptoms. Similarly, methods are needed to differentiate those effects due to the teratogenic insults of alcohol from those that may be related to genetic or environmental factors. Currently, we often associate the consequences of prenatal alcohol exposure with physical outcomes, such as minor facial anomalies, but with further research hopefully this will change and the diagnosis of prenatal alcohol effects will be thought of more in terms of the neurofunctional consequences of such exposure. While the preclinical research has elucidated many of the mechanisms involved in alcohol's teratogenicity, more work is needed to translate these findings into ways to prevent and intervene in the consequences of the alcohol exposure. Finally, while this issue has been primarily devoted to reviewing the consequences of prenatal alcohol exposure, this work also provides guidance on normal brain-behavioral relationships.

\section{Acknowledgments}

The authors greatly acknowledge the help of Jason Dudley and Kristen Breit in preparing the figure for this manuscript. Preparation of this paper was supported by National Institute of Health NIAAA Grants R01 AA010417, U24 AA014811, and T32 AA013525 to epr. and was conducted in conjunction with the Collaborative Initiative on Fetal Alcohol Spectrum Disorders (CIFASD). More information about the CIFASD can be found at www.cifasd.org

\section{References}

Aase JM, Jones K, Clarren SK. Do we need the term "FAE"? Pediatrics. 1995; 95(3):428-430. [PubMed: 7862486] 
Abel EL, Sokol RJ. A revised conservative estimate of the incidence of FAS and its economic impact. Alcoholism: Clinical and Experimental Research. 1991; 15(3):514-524.

Astley, SJ. Diagnostic guide for fetal alcohol spectrum disorders: The 4-digit diagnostic code. 3rd ed.. University of Washington Publication Services; Seattle, WA: 2004. http://depts.washington.edu/ fasdpn/pdfs/guide2004.pdf

Astley SJ, Clarren SK. Diagnosing the full spectrum of fetal alcohol-exposed individuals: Introducing the 4-digit diagnostic code. Alcohol and Alcoholism. 2000; 35(4):400-410. [PubMed: 10906009]

Bertrand, J.; Floyd, RL.; Weber, MK.; O'Connor, M.; Riley, EP.; Johnson, KA., et al. Fetal alcohol syndrome: guidelines for referral and diagnosis. Centers for Disease Control and Prevention; Atlanta, GA: 2004. http://www.cdc.gov/ncbddd/fasd/documents/FAS_guidelines_accessible.pdf

Bertrand J, Floyd RL, Weber MK. Guidelines for identifying and referring persons with fetal alcohol syndrome. Morbidity and Mortality Weekly Report. 2005; 54(RR-11):1-14. [PubMed: 15647722]

Burd L, Hofer R. Biomarkers for detection of prenatal alcohol exposure: a critical review of fatty acid ethyl esters in meconium. Birth Defects Research. Part A, Clinical And Molecular Teratology. 2008; 82(7):487-493.

Burd L, Klug M, Martsolf J. Increased sibling mortality in children with fetal alcohol syndrome. Addiction Biology. 2004; 9(2):179-186. [PubMed: 15223547]

Calhoun F, Warren K. Fetal alcohol syndrome: Historical perspectives. Neuroscience and Biobehavioral Reviews. 2007; 31(2):168-171. [PubMed: 17224346]

Center for Disease Control and Prevention (CDC). Fetal alcohol syndrome-United States, 19791992. Morbidity and Mortality Reports. 1993; 42(17):339-341.

Center for Disease Control and Prevention (CDC). Update: Trends in fetal alcohol syndrome-United States, 1979-1993. Morbidity and Mortality Reports. 1995; 44(13):249-51.

Center for Disease Control and Prevention (CDC). Surveil-lance for fetal alcohol syndrome using multiple sources- Atlanta, Georgia, 1981-1989. Morbidity and Mortality Reports. 1997; 46(47): 1118-20.

Center for Disease Control and Prevention (CDC). Fetal alcohol syndrome-Alaska, Arizona, Colorado, and New York, 1995-1997. Morbidity and Mortality Reports. 2002; 51(20):433-435.

Chudley AE, Conry J, Cook JL, Loock C, Rosales T, LeBlanc N. Fetal alcohol spectrum disorder: Canadian guidelines for diagnosis. Canadian Medical Association Journal. 2005; 172(5 Suppl):S1S21. [PubMed: 15738468]

Clarren SK, Smith DW. The fetal alcohol syndrome. The New England Journal of Medicine. 1978; 298(19):1063-1067. [PubMed: 347295]

Clarren SK, Chudley AE, Wong L, Friesen J, Brant R. Normal distribution of palpebral fissure lengths in Canadian school age children. Canadian Journal of Clinical Pharmacology. 2010; 17:e67-e78. [PubMed: 20147771]

Datta S, Turner D, Singh R, Ruest LB, Pierce WM Jr. Knudsen TB. Fetal alcohol syndrome (FAS) in C57BL/6 mice detected through proteomics screening of the amniotic fluid. Birth Defects Research Part A, Clinical and Molecular Teratology. 2008; 82(4):177-186.

Garcia-Algar O, Kulaga V, Gareri J, Koren G, Vall O, Zuccaro P, et al. Alarming prevalence of fetal alcohol exposure in a Mediterranean city. Therapeutic Drug Monitoring. 2008; 30(2):249-254. [PubMed: 18367990]

Gareri J, Lynn H, Handley M, Rao C, Koren G. Prevalence of fetal ethanol exposure in a regional population-based sample by meconium analysis of fatty acid ethyl esters. Therapeutic Drug Monitoring. 2008; 30(2):239-45. [PubMed: 18367988]

Gifford AE, Farkas KJ, Jackson LW, Molteno CD, Jacobson JL, Jacobson SW, et al. Assessment of benefits of a universal screen for maternal alcohol use during pregnancy. Birth Defects Research. Part A, Clinical and Molecular Teratology. 2010; 88(10):838-846.

Goh YI, Hutson JR, Lum L, Roukema H, Gareri J, Lynn H, et al. Rates of fetal alcohol exposure among newborns in a high-risk obstetric unit. Alcohol (Fayetteville, N.Y.). 2010; 44(7-8):629634.

Goodlett CR, Horn KH, Zhou FC. Alcohol teratogenesis: mechanisms of damage and strategies for intervention. Experimental Biology And Medicine (Maywood, N.J.). 2005; 230(6):394-406. 
Hall JG. New palpebral fissure measurements. American Journal of Medical Genetics Part A. 2010; 152A:1870. [PubMed: 20583189]

Hall, JG.; Froster-Iskenius, UG.; Allanson, JE. Handbook of Normal Physical Measurements. Oxford University Press; New York: 1989.

Harlap S, Shiono PH. Alcohol, smoking, and incidence of spontaneous abortions in the first and second trimester. Lancet. 1980; 2:173-176. [PubMed: 6105340]

Hoyme HE, May PA, Kalberg WO, Kodituwakku P, Gossage JP, Trujillo PM, et al. A practical clinical approach to diagnosis of fetal alcohol spectrum disorders: clarification of the 1996 Institute of Medicine criteria. Pediatrics. 2005; 115(1):39-47. [PubMed: 15629980]

Hutson J, Magri R, Gareri J, Koren G. The incidence of prenatal alcohol exposure in Montevideo Uruguay as determined by meconium analysis. Therapeutic Drug Monitoring. 2010; 32(3):311317. [PubMed: 20445483]

Jones KL, Smith DW. Recognition of the fetal alcohol syndrome in early infancy. Lancet. 1973; 2(7836):999-1001. [PubMed: 4127281]

Jones KL, Smith DW, Ulleland CN, Streissguth AP. Pattern of malformation in offspring of chronic alcoholic mothers. Lancet. 1973; 1(7815):1267-1271. [PubMed: 4126070]

Jones KL, Smith DW, Hanson JW. The fetal alcohol syndrome: clinical delineation. Annals of the New York Academy of Sciences. 1976; 273:130-139. [PubMed: 1072341]

Kline J, Shrout P, Stein Z, Susser M, Warburton D. Drinking during pregnancy and spontaneous abortion. Lancet. 1980; 2(8187):176-180. [PubMed: 6105341]

Kulaga V, Shor S, Koren G. Correlation between drugs of abuse and alcohol by hair analysis: parents at risk for having children with fetal alcohol spectrum disorder. Alcohol (Fayetteville, N.Y.). 2010; 44(7-8):615-621.

Lupton C, Burd L, Harwood R. Cost of fetal alcohol spectrum disorders. American Journal of Medical Genetics Part C (Seminars in Medical Genetics). 2004; 127C(1):42-50.

May PA, Gossage JP. Estimating the prevalence of fetal alcohol syndrome: a summary. Alcohol Research and Health. 2001; 25(3):159-167. [PubMed: 11810953]

May PA, Fiorentino D, Gossage JP, Kalberg WO, Hoyme HE, Robinson LK, et al. Epidemiology of FASD in a province in Italy: Prevalence and characteristics of children in a random sample of schools. Alcoholism, Clinical and Experimental Research. 2006; 30(9):1562-1575.

May PA, Gossage JP, Marais A, Adnams CM, Hoyme HE, Jones KL, et al. The epidemiology of fetal alcohol syndrome and partial FAS in a South African community. Drug and Alcohol Dependence. 2007; 88(2-3):259-271. [PubMed: 17127017]

May PA, Gossage JP, Kalberg WO, Robinson LK, Buckley D, Manning M, et al. Prevalence and epidemiologic characteristics of FASD from various research methods with an emphasis on recent in-school studies. Developmental Disabilities Research Reviews. 2009; 15(3):176-192. [PubMed: 19731384]

Olson, HC.; Ohlemiller, MM.; O'Connor, MJ.; Brown, CW.; Morris, CA.; Damus, K.; National Task Force on Fetal Alcohol Syndrome and Fetal Alcohol Effect. A call to action: Advancing Essential Services and Research on Fetal Alcohol Spectrum Disorders-A report of the National Task Force on Fetal Alcohol Syndrome and Fetal Alcohol Effect. Mar. 20092009 www.cdc.gov/ncbddd/fas

Petković G, Barisić I. FAS prevalence in a sample of urban schoolchildren in Croatia. Reproductive Toxicology. 2010; 29(2):237-241. [PubMed: 19922788]

Sampson PD, Streissguth AP, Bookstein FL, Little RE, Clarren SK, Dehaene P, et al. Incidence of fetal alcohol syndrome and prevalence of alcohol-related neurodevelopmental disorder. Teratology. 1997; 56(5):317-326. [PubMed: 9451756]

Sokol RJ, Delaney-Black V, Nordstrom B. Fetal alcohol spectrum disorder. JAMA: The Journal of the American Medical Association. 2003; 290(22):2996-2999. [PubMed: 14665662]

Spohr HL, Steinhausen HC. Fetal alcohol spectrum disorders and their persisting sequelae in adult life. Deutsches Ärzteblatt International. 2008; 105(41):693-698. [PubMed: 19623288]

Stade B, Ali A, Bennett D, Campbell D, Johnston M, Lens C, et al. The burden of prenatal exposure to alcohol: revised measurement of cost. Canadian Journal of Clinical Pharmacolology. 2009; 16(1):e91-e102. 
Stratton, K.; Howe, C.; Battaglia, F. Fetal alcohol syndrome: Diagnosis, epidemiology, prevention, and treatment. National Academy; Washington, DC: 1996.

Thomas LT, Gaitantzis YA, Frias JL. Palpebral fissure length from 29 weeks to 14 years. Journal of Pediatrics. 1987; 111(2):267-268. [PubMed: 3612403] 

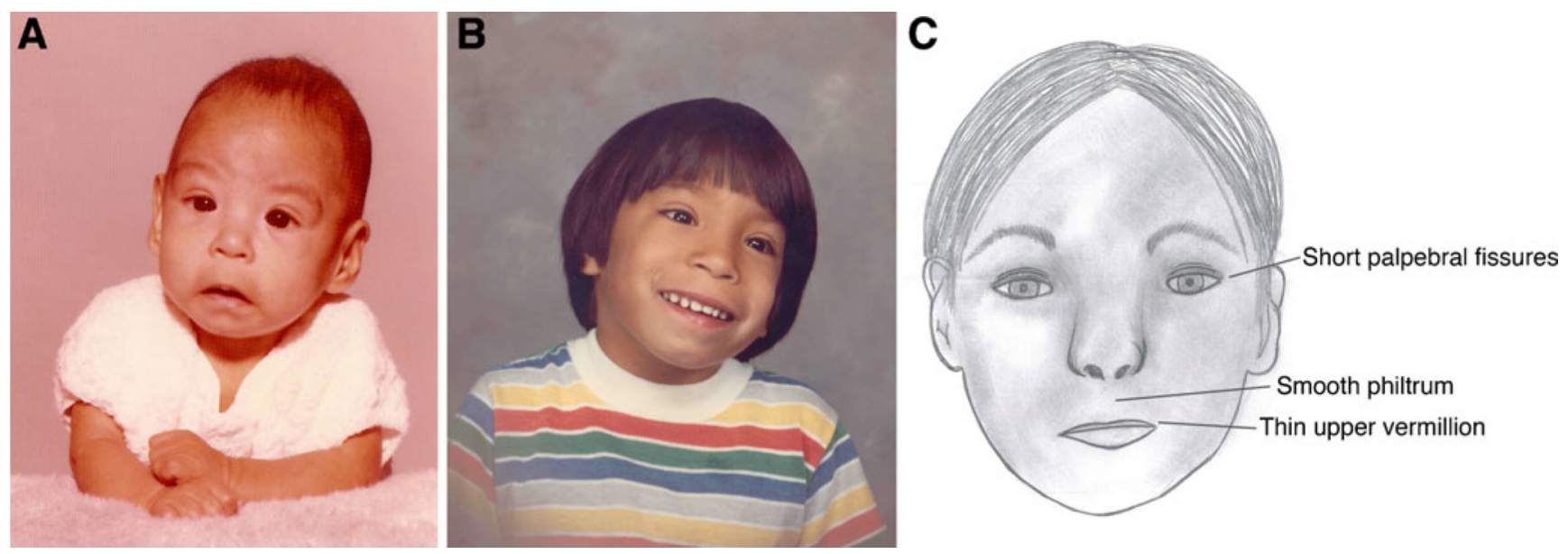

Fig. 1.

a Photo of a 4 month old infant with FAS. b Photo of same child at 5 years of age. c Cartoon showing the major facial characteristics used in the diagnosis of FAS. Photos courtesy of T. Kellerman and used with permission. Original cartoon by Kristen Breit and used with permission 


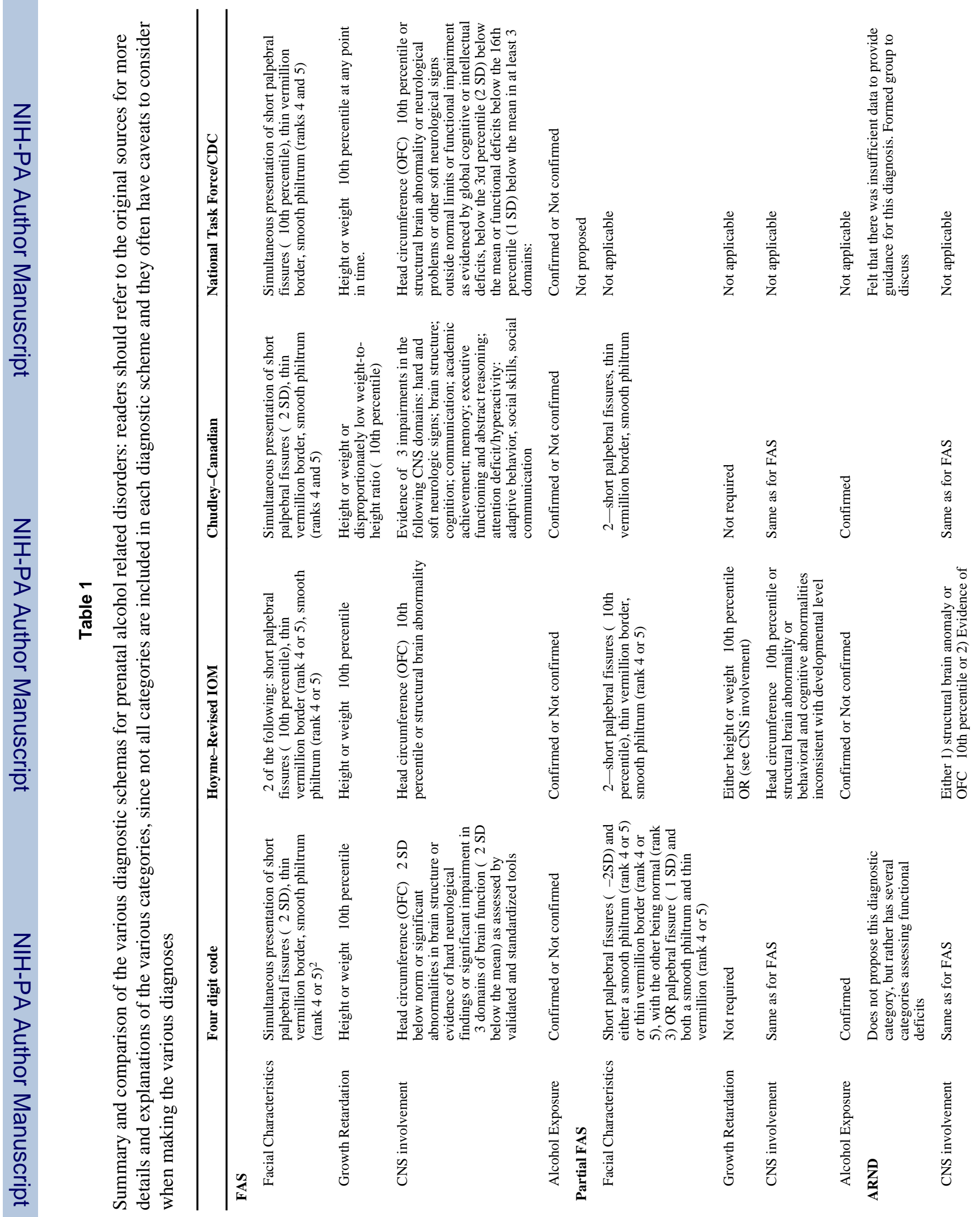




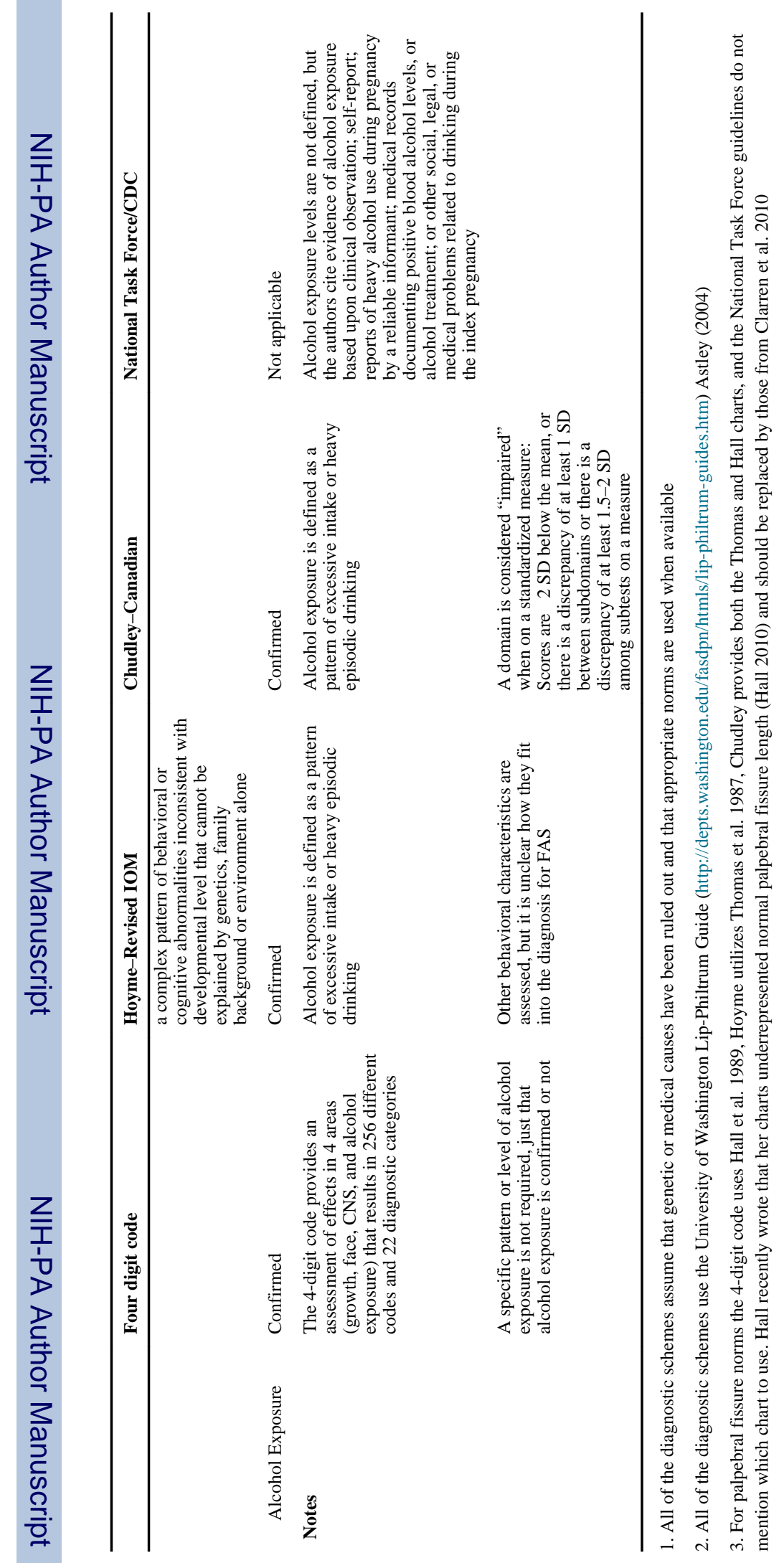

Neuropsychol Rev. Author manuscript; available in PMC 2013 September 20. 